\title{
ANÁLISE POSTURAL: UM ESTUDO SOBRE AS ASSIMETRIAS, DESVIOS POSTURAIS E ESTADO NUTRICIONAL DE ESCOLARES
}

\author{
Paula Ciol, Universidade Paulista - UNIP, Campinas - Brasil \\ Antonia Dalla Pria Bankoff, Faculdade de Educação Física - UNICAMP, Campinas - Brasil \\ Carlos Aparecido Zamai, Faculdade de Educação Física - UNICAMP, Campinas - Brasil
}

\section{RESUMO}

As alterações morfológicas decorrem dos hábitos posturais e são somatizadas ao longo da vida, repercutindo tanto na estática quanto na dinâmica do sistema locomotor, sendo particularmente evidentes em indivíduos predispostos. Essa problemática, quando associada com a má nutrição e desnutrição, assim como sobrepeso corporal, interfere na postura. Neste sentido, o presente estudo teve como objetivo principal analisar as assimetrias e desvios posturais de escolares na faixa etária de 10 a 17 anos de idade de ambos os sexos e verificar sua relação com o estado nutricional, para tanto, realizou-se avaliação postural computadorizada e avaliação antropométrica. Analisando os resultados pode-se observar que tanto os escolares do sexo masculino como do sexo feminino com estado nutricional deficitário apresentaram acentuação considerável da curvatura lordótica lombar. Portanto, a pesquisa demonstrou haver uma relação direta entre a má nutrição, desnutrição e problemas posturais ligado ao sistema locomotor.

Palavras-Chaves: Desvios posturais; Assimetrias; Estado nutricional; Escolares.

\section{POSTURAL ANALYSIS: A STUDY ABOUT POSTURAL ASYMMETRIES AND DEVIATIONS AND NUTRITIONAL STATE OF STUDENTS}

\begin{abstract}
Morphological alterations are caused by postural habits and are somatized during life with repercussions on the static as well as the dynamic position of the locomotive system, being especially evident in predisposed individuals. This issue, when associated with malnutrition and lack of nourishment as well as overweight, interferes with posture. In this sense, the present study had as its primary objective the analysis of postural asymmetries and deviations in students between 10 and 17 years old of both genders as well as the verification of their relationship with the students' nutritional state by means of computerized postural assessment and anthropometric evaluation. In analyzing the results, it was observed that both male and female students with poor nutritional state presented considerable accentuation of lumbar lordotic curvature. The study demonstrated that there is a direct relationship between malnutrition, lack of nourishment and postural problems linked to the locomotive system.
\end{abstract}

Key-Words: Postural deviations; Asymmetries; Nutritional state; Students.

Conexões: revista da Faculdade de Educação Física da UNICAMP, Campinas, v. 10, n. 3, p. 32-41, set./dez. 2012. 


\section{INTRODUÇÃO}

\section{Aspectos Gerais sobre Postura}

Segundo Souchard, ${ }^{1}$ o homem antes de tudo é herdeiro de seus antepassados e deve às vezes suportar eventuais imperfeições que estes apresentaram e que lhe negaram. Da concepção à morte, deve gerir da melhor forma este patrimônio imperfeito, apesar das agressões de todo tipo das quais ele não pode deixar de ser vítima. O meio desempenha um papel especial em seu desenvolvimento. A sociedade lhe impõe condições de vida coerciva. As extraordinárias faculdades de adaptação do homem permitem-lhe, com frequiência acomodar-se em condições horríveis. Mas o homem detém também, em relação aos outros reinos, a superioridade de ter consciência de seu estado, de suas possibilidades, daquilo que o circunda e governa.

Apesar da postura básica de uma pessoa ser natural, influências externas como a disposição, a idade, os esportes, a nutrição, a doença e as condições de vida e de trabalho têm um efeito considerável na mesma. ${ }^{2}$ Ainda a postura de um indivíduo é a somatização de todo o seu passado, seu cotidiano, sua forma de se posicionar diante das situações de lazer, trabalho e de repouso, seu estado emocional entre outros. Neste sentido, durante o transcorrer do dia-a-dia, submetemos nosso corpo a posições variadas consciente e inconscientemente.

A coluna vertebral, suporte do corpo, é a mais prejudicada com sobrecargas que resultam no aumento significativo de problemas posturais da população mundial, tanto em adultos como em crianças. $^{3}$

Com o auxílio da análise postural é possível identificar desvios localizados em diversos segmentos corporais que geralmente trazem conseqüências danosas à função de sustentação e de mobilidade. As alterações, em nível de debilidades, revelam-se de grande incidência entre escolares nas faixas etárias de $1^{\circ}$ grau. ${ }^{4-7}$

\section{Nutrição X Desnutrição}

Os dois problemas relacionados à alimentação são a má nutrição e a desnutrição, ou seja, não saber comer e não ter o que comer. Por isso, tanto a má nutrição quanto à desnutrição, causam prejuízos à saúde.

Conexões: revista da Faculdade de Educação Física da UNICAMP, Campinas, v. 10, n. 3, p. 32-41, set./dez. 2012. 
O ser humano necessita ingerir determinados tipos e quantidades de nutrientes para ser considerado saudável e quando essa ingestão de nutrientes não consegue alcançar o ideal para suprir os requerimentos metabólicos do organismo, ocorre a desnutrição. A desnutrição leva a uma série de alterações na composição corporal e no funcionamento normal do organismo, quanto mais grave for o caso, maiores e também mais graves serão as repercussões orgânicas. Uma população desnutrida representa maiores gastos em saúde para o país, desde os cuidados primários até a internação. ${ }^{8}$

\section{Obesidade}

Atualmente a obesidade na infância e adolescência tem adquirido proporções epidêmicas, consta no Brasil que a obesidade está em torno de $20 \%$ segundo informações do Ministério da Saúde. 9

Todo um sistema de vida inadequado provavelmente favoreça esse tipo de acontecimento: sedentarismo, hábitos familiares inadequados, alimentação insatisfatória, excesso de carboidratos na dieta, a velocidade da refeição, os lanches desequilibrados e o consumo de doces e guloseimas. Segundo Collipp ${ }^{10}$ o ritmo de armazenamento não é o mesmo para as várias idades e etapas do crescimento, devendo ainda ser consideradas as diferenças sexuais. Freqüentemente as meninas possuem mais tecido gorduroso que os meninos durante o estirão puberal, aspecto este que acentua na adolescência.

Portanto, levando em consideração a problemática da má nutrição, desnutrição ${ }^{8}$ assim como sobrepeso corporal verifica-se a interferência destes na postura, além da influência dos hábitos posturais inadequados que são somatizadas ao longo da vida. ${ }^{11-12}$

\section{OBJETIVOS}

\section{Objetivo Geral}

Estudar através da biomecânica corporal, as assimetrias e desvios posturais e suas relações com o estado nutricional em crianças e adolescentes na faixa etária de 10 a 17 anos de idade de do município de Votuporanga/São Paulo.

\section{Objetivos Específicos}

Conexões: revista da Faculdade de Educação Física da UNICAMP, Campinas, v. 10, n. 3, p. 32-41, set./dez. 2012. ISSN: $1983-9030$ 
- Identificar as assimetrias e desvios posturais através da avaliação postural computadorizada;

- Identificar o índice de massa corporal (IMC) através das medidas antropométricas;

- Relacionar as assimetrias e desvios com o índice de massa corporal.

\section{MATERIAL E MÉTODO}

\section{Sujeitos}

Foi avaliado um total de 71 sujeitos de ambos os sexos (55 meninas e 16 meninos), na faixa etária de 10 a 17 anos de idade, todos estudantes do ensino fundamental de $5^{\text {a }}$ a $8^{\text {a }}$ séries, da Escola Estadual "Sebastião Almeida de Oliveira", localizada no Bairro São Cosme, periferia do município de Votuporanga/São Paulo, escola escolhida aleatoriamente.

\section{Universo da Pesquisa}

A Escola "Sebastião Almeida de Oliveira", localizada no Bairro São Cosme, periferia do município de Votuporanga, possui apenas o Ensino Fundamental, ou seja, de $1^{\mathrm{a}}$ a $8^{\mathrm{a}}$ séries, número limitado de salas de aulas, carteiras com assento duplo, antiergonômica e total de carteiras inferior ao número de alunos constante em salas de aulas, desprovida de espaços para prática de aulas de educação física, mantendo improvisações inadequadas para esta prática de aula, com infra-estrutura pobre e precária, com falta de material de todos os gêneros. Quanto à nutrição, ou seja, merenda escolar fornecida pelo estado, apresenta-se pobre em vitaminas, proteínas e sais minerais, deixando os alunos sempre em situação de carência nutricional, sendo que a maioria deles, não faz outra refeição em suas casas, e os que fazem também consomem alimentos pobres em proteínas, vitaminas e sais minerais, causando insuficiência nutricional.

A montagem dos equipamentos foi feita em sala disponível, localizada na própria escola, onde os

alunos foram examinados e avaliados individualmente. A equipe retornou à escola para informação de pais, alunos e professores bem como, a própria direção, dos resultados obtidos na pesquisa.

\section{MATERIAL UTILIZADO NA COLETA DOS DADOS}

Foi realizado o exame biométrico (nível antropométrico), através de peso corporal e da estatura de cada aluno, com o objetivo de estudar as relações nutricionais com as alterações morfológicas 
posturais. Para tanto foi utilizado uma balança antropométrica Filizola com toesa, sendo a aferição do peso averiguada em $\mathrm{Kg}$ com capacidade de até $150 \mathrm{~kg}$ e a estatura em metros com casas centesimais.

Com a finalidade de obter-se o nível de assimetrias e desvios posturais dos alunos que participaram desta pesquisa, foi utilizado um sistema de avaliação postural computadorizada (Software Análise Postural DaVinci - versão 3.0.3.100) desenvolvido pela Micromed Biotecnologia, pertencente ao Laboratório de Eletromiografia e Avaliação Postural da Universidade Estadual de CampinasUnicamp, adquirido através do projeto Auxílio Integrado a Pesquisa CNPq/FAPESP número 52.0006/96, o qual conta com um programa específico para avaliação postural. É um sistema que possibilita a captura de imagens e medidas de ângulos e distâncias da postura humana. Constituído por uma placa de aquisição de sinais de vídeo e um programa que possibilita a visualização das fotos em um único monitor colorido de média resolução (640 x 480 pontos) com a opção de SVGA (800 x 600) ou (1024 x 768) pontos, com tela de 14 polegadas ou superior. Foi utilizada uma filmadora Sharp, conectada ao computador gravando todos os dados da avaliação postural. O uso de um fio de prumo e de uma fita de calibração possibilitou que em todas as fotos fosse obtida uma referência absoluta para a precisão das medidas.

\section{COLETA DE DADOS}

A investigação foi iniciada com o exame biométrico a nível antropométrico, através do peso corporal e da estatura de cada aluno.

Peso e estatura: O peso e a estatura dos sujeitos envolvidos na pesquisa foram obtidos através de uma balança Filizola com toesa, sendo a aferição do peso averiguada em Kg e a estatura em metros.

Avaliação Postural Computadorizada: Com vista à análise das assimetrias e desvios posturais dos alunos, todos se apresentaram no local vestidos com um calção de banho, no caso masculino, e maiô de duas peças no caso feminino. Posteriormente foram demarcados com etiquetas auto-adesivas, os pontos anatômicos de referência para a coleta de dados.

Os pontos são identificados a seguir:

Conexões: revista da Faculdade de Educação Física da UNICAMP, Campinas, v. 10, n. 3, p. 32-41, set./dez. 2012. 


\section{Plano Frontal - Vista Dorsal:}

- Ponto acromial projetada $3 \mathrm{~cm}$ para baixo, em direção a escápula;

- Ângulo inferior das escápulas;

- Olécranos;

- Linha Glútea

- Linha Poplítea;

- Calcâneo;

\section{Plano Sagital-Vista Lateral esquerda:}

- Meato auditivo externo projetado $3 \mathrm{~cm}$ a frente;

- Epicôndilo lateral;

- Maléolo lateral

- Acrômio, projetado a $3 \mathrm{~cm}$ em direção ao úmero

- Olecrano.

Após a demarcação dos pontos anatômicos de referência, os alunos adotaram uma postura ortostática estática seguindo um ponto referencial demarcado no solo, o mais natural possível, com os pés simetricamente colocados sobre essa demarcação e os calcanhares ligeiramente afastados, seguindo as instruções do manual da Micromed Biotecnologia. A filmadora ficou apoiada num tripé a uma altura de $1,20 \mathrm{~m}$ e distância de 3,10m da demarcação e em seguida foi efetuada a coleta de dados.

\section{APRESENTAÇÃO DOS RESULTADOS E DISCUSSÃO}

Para uma maior interpretação e compreensão dos resultados, os mesmos serão apresentados na Tabela 1.

Tabela 1 - Dados referentes à classificação do IMC segundo a OMS, \% (percentual) da população (feminino e masculino) e distribuição das médias gerais das assimetrias e desvios posturais medidos em ângulos, dos escolares 


\begin{tabular}{lccccc}
\hline Classificação do IMC \% & \multicolumn{5}{c}{ Desvios e Assimetrias (em graus) } \\
& \multicolumn{5}{c}{ Sexo Feminino } \\
& & Cifose & Lordose & Lordose & Ombros \\
& Dorsal & Cervical & Lombar & Caídos \\
\hline Baixo peso & 41.81 & -25.59 & -26.87 & 17.82 & 0.13 \\
Peso Normal & 47.27 & -26.82 & -29.56 & 13.94 & -0.16 \\
Sobrepeso & 9.09 & -28.09 & -28.34 & 12.62 & 1.8 \\
Obesidade II & 1.81 & -27.15 & -27.15 & 14.06 & 1.49 \\
\hline & Desvios e Assimetrias (em graus) & \\
\hline Baixo peso & 75.0 & -24.55 & -18.72 & 17.94 & 1.84 \\
Peso Normal & 18.75 & -27.39 & -23.84 & 18.11 & -0.51 \\
Sobrepeso & 6.25 & -31.13 & -30.23 & 9.64 & -0.85 \\
\hline
\end{tabular}

O IMC (Índice de Massa Corporal) é um padrão internacional de cálculo da obesidade de um indivíduo adotado pela OMS (Organização Mundial da Saúde). Os valores preconizados pela OMS são: 18,5 abaixo de peso; 18,5 a 25 considerado peso normal; 25 a 30 acima do peso e acima de 30 obeso. Conforme os dados apresentados na tabela 01, em relação ao índice e massa corporal-IMC, $41,81 \%$ das meninas e $75 \%$ dos meninos apresentaram IMC abaixo do peso. Segundo a OMS resultados abaixo deste índice não são saudáveis e são preocupantes. Na faixa recomendável segundo a OMS (18,5 a 25-IMC) as meninas apresentaram percentual de 47,27\% e os meninos apresentaram percentual muito baixo, ou seja, apenas $18,75 \%$ estão na faixa recomendável. É interessante observar que nos meninos o sobrepeso é menor em percentual e não foi encontrado obesidade, muito embora o número de participantes da pesquisa do sexo feminino foi universo maior. Portanto, em relação ao IMC os resultados preocupantes são o sobrepeso nas meninas que registrou $9,09 \%$ e baixo peso nos meninos de $75 \%$.

Quanto às assimetrias e desvios posturais medidos em ângulos corporais relacionados ao índice de massa corporal, observamos que nos escolares de ambos os sexos classificados como sobrepeso evidenciaram-se as maiores angulações nas curvaturas cifótica dorsal e lordótica cervical, porém, os valores apresentados pela população do sexo feminino com obesidade tipo II foram menores. Considerando os aspectos anatômicos e cinesiológicos do corpo humano, em relação ao peso 
corporal, há evidências que ele, especificamente no abdome, demonstra maior a probabilidade de desenvolver curvaturas acentuadas na coluna vertebral. ${ }^{13}$

Das escolares do sexo feminino, 47,27\% encontram-se na faixa recomendável de peso, apresentando, no entanto, acentuada curvatura lordórtica cervical. A maior angulação da curvatura lordótica lombar também foi evidenciada nas escolares com baixo peso, demonstrando haver uma relação direta entre má nutrição, desnutrição e problemas posturais ligado ao sistema locomotor.

De modo geral, considerando os resultados, observamos que as meninas apresentaram lordose cervical, com projeção proeminente para frente do pescoço, muito mais que os meninos, com exceção para o sobrepeso. Um dos fatores que Tribastone ${ }^{12}$ aponta é que as meninas nesta faixa etária procuram esconder a formação dos seios, abrindo/alando mais as escápulas, no sentido de proteção. Isso, conseqüentemente, por estarem na fase de crescimento e desenvolvimento, provoca alterações morfológicas da idade evolutiva em toda a coluna vertebral, especificamente lordose bastante acentuada, alterando a morfologia do abdome, ou seja tornando-a mais proeminente e na maioria das vezes provocando desalinhamento nos membros inferiores.

O fator alimentar/nutricional é parceiro importante do sistema locomotor, ou seja, toda deficiência nutricional somativa da primeira infância, começa aparecer sobre o mesmo, então, existe uma associação inegável entre nutrição/alimentação e postura corporal, tornando evidente no sexo masculino, o qual nas medidas antropométricas, apresentou estarem muito mais abaixo do peso, quando comparados com as meninas. A preocupação existente é exatamente a questão nutricional oferecida às escolas pertencentes ao Estado, ou seja, aquelas não municipalizadas que se referem ao ensino de $5^{\mathrm{a}}$ a $8^{\mathrm{a}}$ séries. As prefeituras, por sua vez, não reforçam o alimento recebido do Estado que é repassado sem teor nenhum de proteínas, sais minerais e vitaminas, sendo em sua maior parte, carboidratos.

Além das deficiências encontradas, também, observamos que nenhuma disciplina ministrada na escola tem a preocupação em realizar uma análise da situação antropométrica e postural, pelo contrário, parece ser temas muito distantes da realidade vivenciada pelos professores daquela escola, muito embora, as disciplinas de educação física escolar e ciências possuem conhecimentos para a 
realização de um trabalho mais efetivo junto aos escolares em relação às temáticas discutidas neste trabalho.

\section{CONCLUSÃO}

O presente trabalho, através dos resultados apresentados evidenciou estreita relação entre os fatores alimentar e nutricional com o desenvolvimento do sistema locomotor e sua importância para a postura corporal na faixa etária estudada. Encontramos resultados preocupantes em relação ao sexo masculino no que se refere ao percentual de índice de massa corporal abaixo do peso constituindo percentual de 75\%. Em contrapartida, foi evidenciado percentual maior de sobrepeso no sexo feminino e também obesidade, não sendo registrada obesidade no sexo masculino.

Quando se buscou relacionar os desvios posturais e as assimetrias posturais encontramos: na classificação do IMC - sobrepeso foi encontrado valores maiores de angulações referentes aos desvios posturais cifose dorsal e lordose cervical; a maior angulação da curvatura lordótica lombar foi evidenciada em escolares do sexo feminino com baixo peso. Em relação aos ombros caídos os maiores valores foram encontrados na classificação do IMC- sobrepeso e obesidade no sexo feminino e baixo peso no sexo masculino.

\section{SUGESTÃO}

Ficou demonstrado, que há necessidade de debates e discussão, bem como, explanação sobre postura, hábitos posturais adequados tanto na escola como fora dela, assim como suas relações com a nutrição conscientizando professores, pais, alunos e prefeituras, sobre alimentação adequada para crianças e adolescentes nesta faixa etária.

\section{REFERÊNCIAS}

${ }^{1}$ SOUCHARD, P. E. Reeducação postural global. 2. ed. São Paulo: Ícone, 1986.

${ }^{2}$ EITNER, D.et al. Fisioterapia nos esportes. São Paulo: Manole, 1984.

${ }^{3}$ BRACCIALLI, L. M.; VILARTA, R. Postura corporal: reflexões teóricas. Revista Fisioterapia em Movimento, v. 14, n. 1, abr./set. 2001. 
${ }^{4}$ INGELMARK, B. E. The influence of different forms of physical edication upon the posture in childhood. Fiep Bulletin, v. 3, n. 2/3, p. 18-26, 1953.

${ }^{5}$ CLARKE, H. Posture phsicalfiness. Séries, v. 9, n. 1, 1979.

${ }^{6}$ BRIGHETTI, V.; BANKOFF, A. D. P. Levantamento de incidência de cifose postural e ombros caídos em alunos de $1^{\circ}$ à $4^{\circ}$ séries escolar. Revista Brasileira de Ciências do Esporte, v. 7, n. 3, p. 93-97, 1986.

${ }^{7}$ BRIGHETTI, V. Avaliação postural em escolares das redes estadual e particular de ensino de primeiro grau. 1993. 95 f. Dissertação (Mestrado em Educação Física) - Faculdade de Educação Física, Universidade Estadual de Campinas, Campinas, 1993.

${ }^{8}$ INSTITUTO NACIONAL DE ALIMENTAÇÃO E NUTRIÇÃO (INAN). Pesquisa nacional sobre saúde e nutrição: resultados preliminares. 2. ed. Brasília: Ministério da Saúde, 1990.

${ }^{9}$ BRASIL. Ministério da Saúde. Programa de Pesquisa Nacional sobre Nutrição. BRASÍLIA, 1999.

${ }^{10}$ COLLIPP, P. J. Tratamento de criança obesa. São Paulo: Manole, 1977.

${ }^{11}$ BANKOFF, A. D. P. et al. Postura corporal. In: CONGRESSO CATARINENSE DE MEDICINA DESPORTIVA, 1. Florianópolis, 1993. Anais... Florianópolis, 1993.

${ }^{12}$ TRIBASTONE, F. Tratado de exercícios corretivos aplicados à reeducação motora postural. São Paulo: Manole, 2001.

${ }^{13}$ BANKOFF, A. D. P. Morfologia e cinesiologia aplicada ao movimento corporal. Rio de Janeiro: Guanabara Koogan, 2007. 